\title{
Arterial Spin Labeling Measurements of Cerebral Perfusion Territories in Experimental Ischemic Stroke
}

\author{
Renata F. Leoni • Fernando F. Paiva • Byeong-Teck Kang • Erica C. Henning • \\ George C. Nascimento • Alberto Tannús • Draulio B. de Araujo • Afonso C. Silva
}

Received: 5 August 2011 /Revised: 7 October 2011 /Accepted: 11 October 2011 /Published online: 16 November 2011

(C) Springer Science+Business Media, LLC (outside the USA) 2011

\begin{abstract}
Collateral circulation, defined as the supplementary vascular network that maintains cerebral blood flow (CBF) when the main vessels fail, constitutes one important defense mechanism of the brain against ischemic stroke. In the present study, continuous arterial spin labeling (CASL) was used to quantify CBF and obtain perfusion territory maps of the major cerebral arteries in spontaneously hypertensive rats (SHRs) and
\end{abstract}

Renata F. Leoni and Fernando F. Paiva contributed equally to this work

R. F. Leoni $\cdot$ F. F. Paiva $\cdot$ B.-T. Kang $\cdot$ G. C. Nascimento $\cdot$

A. C. Silva $(\triangle)$

Cerebral Microcirculation Unit, Laboratory of Functional and Molecular Imaging, National Institute of Neurological Disorders and Stroke, National Institutes of Health,

10 Center Drive MSC 1065, Building 10 Room B1D106,

Bethesda, MD 20892-1065, USA

e-mail: SilvaA@ninds.nih.gov

\section{R. F. Leoni}

Departamento de Física, FFCLRP,

Universidade de São Paulo,

Ribeirao Preto, Sao Paulo 14040-901, Brazil

F. F. Paiva $\cdot$ A. Tannús

Departamento de Física e Informática, IFSC,

Universidade de São Paulo,

São Carlos, São Paulo 13560-970, Brazil

E. C. Henning

Section on Stroke Diagnostics and Therapeutics,

National Institute of Neurological Disorders and Stroke,

National Institutes of Health,

10 Center Drive MSC 1063, Building 10 Room B1D733,

Bethesda, MD 20892-1063, USA

\section{B. de Araujo}

Instituto do Cérebro, Hospital Universitário Onorio Lopes,

Universidade Federal do Rio Grande do Norte - UFRN,

Natal, Rio Grande do Norte 590560-450, Brazil their normotensive Wistar-Kyoto (WKY) controls. Results show that both WKY and SHR have complementary, yet significantly asymmetric perfusion territories. Right or left dominances were observed in territories of the anterior (ACA), middle and posterior cerebral arteries, and the thalamic artery. Magnetic resonance angiography showed that some of the asymmetries were correlated with variations of the ACA. The leptomeningeal circulation perfusing the outer layers of the cortex was observed as well. Significant and permanent changes in perfusion territories were obtained after temporary occlusion of the right middle cerebral artery in both SHR and WKY, regardless of their particular dominance. However, animals with right dominance presented a larger volume change of the left perfusion territory $(23 \pm 9 \%)$ than animals with left dominance $(7 \pm 5 \%$, $P<0.002)$. The data suggest that animals with contralesional dominance primarily safeguard local $\mathrm{CBF}$ values with small changes in contralesional perfusion territory, while animals with ipsilesional dominance show a reversal of dominance and a substantial increase in contralesional perfusion territory. These findings show the usefulness of CASL to probe the collateral circulation.

Keywords Cerebral blood flow Collateral flow $\cdot$ Magnetic resonance imaging $\cdot$ Vascular territory

\section{Introduction}

Stroke is the third leading cause of death in the USA, affecting 800,000 people and killing about 137,000 people each year. It is a leading cause of long-term disability for adults and the elderly, with an estimated total cost of US $\$ 73.7$ billion in 2010 (source: Stroke.org). Therefore, it is 
imperative to pursue research aimed at preventing the causes of stroke, alleviating its symptoms and minimizing its consequences to the patient.

One important defense mechanism that the brain has to protect itself from a stroke is the collateral circulation, defined as the supplementary vascular network that maintains cerebral blood flow (CBF) when the main vessels fail [1]. The collateral circulation plays a compensatory role in providing an alternative route for blood to reach brain tissue when the principal supply or drainage is compromised. The collateral circulation is composed of anastomoses between segments of the brain vasculature, and vessels of all types (arteries, arterioles, capillaries, venules, and veins) display anastomoses. The primary collateral circulation consists of the arterial segments of the Circle of Willis, which immediately reroute CBF to ischemic regions through their existing anastomoses [1]. Along with the ophthalmic artery, the leptomeningeal circulation contributes to a secondary network of collaterals [1]. Therefore, the collateral circulation, a powerful defense mechanism to safeguard viable tissue and its patency, along with a quantitative assessment of resting perfusion, are two important factors in determining stroke outcome [2-4].

Neuroimaging methods including CT angiography and magnetic resonance imaging (MRI) have had a significant impact in the ability to visualize and quantify collateral circulation [5]. In particular, quantitative MRI of CBF can be performed using arterial spin labeling (ASL) [6-8]. Besides superiority in spatial and temporal resolution when compared to other techniques for perfusion measurement [9, 10], ASL methods are completely non-invasive since they use endogenous arterial water as tracer. Shortly after the inception of ASL came the interest to selectively label blood flowing through specific cerebral feeding arteries [11]. The ability to assess the perfusion territories of major cerebral arteries can be valuable to further understand the collaterals. Maps of vascular territories can provide complementary information to angiography, allowing the verification of the extent of the ischemic region and the flow redistribution resulting from compensatory collateral flow [12]. Several ASL-based methods have been proposed to measure cerebral perfusion territories in humans [12, 13], assessing, for instance, the collateral circulation and alterations in individual vascular territories in atherosclerotic disease [14, 15], in ischemic stroke [16, 17], and in Moyamoya's disease [18].

In the present study, ASL was used to quantify CBF and obtain the perfusion territory maps of major cerebral feeding arteries in the spontaneously hypertensive rat (SHR), a widely used experimental model in the study of stroke and other cerebrovascular diseases [19]. Normotensive Wistar-Kyoto (WKY) rats were used as controls. Magnetic resonance angiography (MRA) was used to assess variations in the formation of the Circle of Willis (COW) that are related to the vascular perfusion territories. In addition, resting $\mathrm{CBF}$ and alterations in perfusion territories were assessed in animals subjected to temporary middle cerebral artery occlusion.

\section{Materials and Methods}

\section{Animal Preparation}

All experiments were approved by the NINDS/NIDCD Animal Care and Use Committee, National Institutes of Health. Male adult Wistar-Kyoto rats (WKY, $N=13$ ) and spontaneously hypertensive rats (SHRs, N=13) (Harlan Laboratories, Somerville, NJ, USA), body weight $297 \pm$ $62 \mathrm{~g}$, were initially anesthetized with isoflurane $(5 \%$ induction, 2\% maintenance), and ventilated with oxygenenriched air. Animals were secured to an MRI-compatible stereotaxic head frame by earpieces and a bite bar. Once animals were in the magnet, heart rate, respiration rate, oxygen saturation $\left(\mathrm{SPO}_{2}\right)$, and end-tidal $\mathrm{CO}_{2}\left(\mathrm{EtCO}_{2}\right)$ were monitored continuously by a pulse oximeter (Surgivet, Waukesha, WI, USA). Rectal temperature was also monitored and maintained at $37 \pm 1^{\circ} \mathrm{C}$ using a heated water blanket.

Rats were separated into two experimental groups. In group 1 (six WKY controls, six SHRs), whole-brain MRI measurements of $\mathrm{CBF}$ and mapping of the perfusion territories of the main cerebral arteries were performed using the continuous ASL (CASL) technique as described below. In addition, MRA of the cerebral arterial vasculature were obtained and correlated to the perfusion territories. In group 2 (seven WKY, seven SHR), rats were subjected to temporary occlusion of the middle cerebral artery occlusion (MCAO), as described below. Whole-brain measurements of $\mathrm{CBF}$ and mapping of perfusion territories were performed before and during $\mathrm{MCAO}$, and again $1 \mathrm{~h}$ and 1 day after reperfusion.

\section{Cerebral Ischemia}

Focal cerebral ischemia was induced temporarily in the rats of group 2 using the intraluminal approach to occlude the right middle cerebral artery (MCA), as previously described [20]. Briefly, the rats were prepared as described above and placed in the MRI scanner for measurements of CBF and of perfusion territories. At the end of the MRI session, the animal was brought back to the operating table and placed in the supine position. The ventral aspect of the neck was shaved and cleaned aseptically. A midline incision was made to expose the right common carotid artery (CCA) and the carotid bulb. The right external carotid artery (ECA) 
was transected and the internal carotid artery (ICA) was carefully isolated. The CCA was clamped, and a 4-0 silicone-coated nylon suture (Doccol Corporation, Redlands, CA, USA) was inserted through the ECA stump into the right ICA until resistance was felt due to lodging of the tip of the suture at the bifurcation of the ICA with the MCA and the anterior cerebral artery (ACA) at the Circle of Willis [20]. Immediately after occlusion, the CCA clamp was removed, all wounds were closed and the animal was taken to the MRI for measurements of $\mathrm{CBF}$ and of perfusion territories. No other vessels were transected or occluded. At the end of the MRI session, which typically lasted 35-40 min, the animal was removed from the scanner and brought back to the operating table. At $1 \mathrm{~h}$ after MCAO, the inserted suture was withdrawn to permit reperfusion. The suture was then fully removed from the ICA, and the ECA stump was tightly sutured to prevent bleeding. All wounds were treated with $1 \%$ lidocaine and sutured closed. The animal was then returned to the magnet a third time for subsequent MRI measurements.

\section{Arterial Spin Labeling Scheme for Measuring Perfusion Territories}

The concept used to obtain selective labeling of individual arteries is illustrated in Fig. 1. This is achieved by combining the limited excitation profile of a dedicated labeling coil, placed underneath the neck of the animal at a distance $\Delta z_{0}$ from the isocenter and an oblique labeling plane. The labeling plane is defined by rotating the labeling gradient, $G_{\text {eff }}$, in the horizontal plane by an angle $\theta$ with respect to the longitudinal axis. Due to the confined excitation profile of the labeling coil, only the arteries passing over the intersection of the labeling plane with the labeling coil transversal plane undergo the ASL inversion. When no transverse gradient is used $(\theta=$ $0^{\circ}$ ), a regular CASL experiment is carried out and both left and right CCAs and vertebral arteries (VAs) are inverted. To guarantee the validity of the conditions for efficient flow-driven adiabatic inversion, the labeling gradient in the $z$-direction was optimized to provide the best labeling efficiency $[8,21]$ and kept constant in all experiments. Thus, the angle $\theta$ was defined simply by the addition of a gradient in the $x$-direction. When $\theta$ is large enough, only the right $\left(\theta>0^{\circ}\right)$ or the left $\left(\theta<0^{\circ}\right)$ arteries are contained in the intersection of the labeling plane with the labeling coil, while the arteries located on the opposite side are intersected by the labeling plane far away from, and thus, out of reach of the labeling coil (Fig. 1a). As recently shown [12], the minimum angle necessary to selectively label the desired artery depends on the effective longitudinal excitation profile of the labeling coil, $E P_{Z}$, and the distance $\delta=\delta_{L}+\delta_{R}$ between the

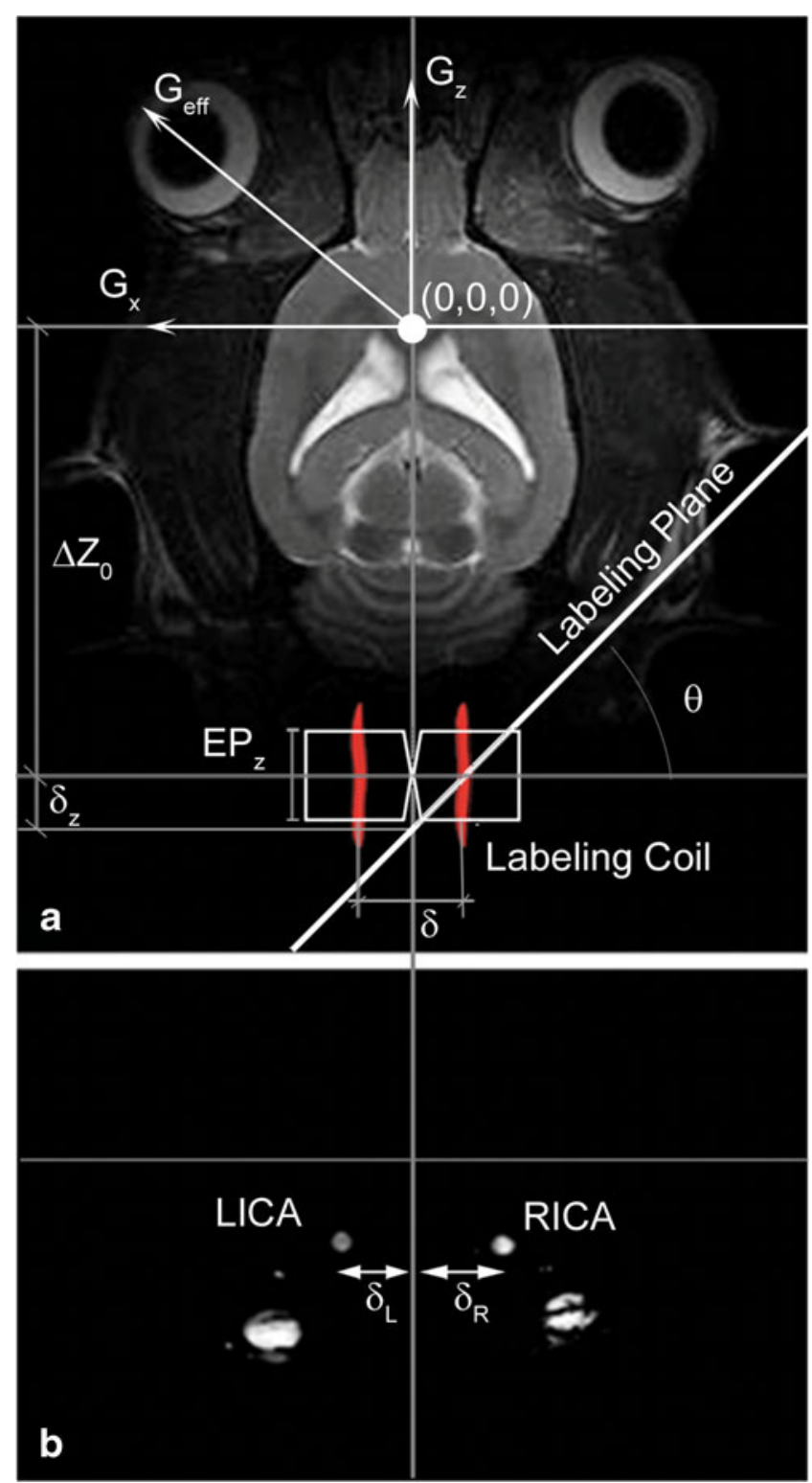

Fig. 1 a Geometrical diagram of the scheme used to selectively label blood flowing in the major cerebral feeding arteries. A dedicated labeling coil is placed under the animal's neck at a distance $\Delta Z_{0}$ from the magnet isocenter. The labeling plane is rotated about the $\mathrm{Y}$ magnet axis by an angle $\theta$ to label only the right $\left(\theta>0^{\circ}\right)$ or the left $\left(\theta<0^{\circ}\right)$ internal carotid artery (ICA). b Cross-sectional image of the neck showing the arteries used to plan the selective labeling scheme. LICA left ICA, RICA right ICA

arteries on opposite sides, depicted in Fig. 1b. The ASL labeling frequency offset considering the tilt in the labeling plane is given by:

$f_{\mathrm{ASL}}=\gamma \cdot G_{z} \cdot\left(\Delta z_{0}+\delta_{z}\right)$

where $\delta_{z}$ is an offset in the longitudinal direction calculated from $\delta_{R}$ or $\delta_{L}$ according to:

$\delta_{z}=\chi \tan \theta, \chi=\left\{\begin{array}{cccc}\delta_{R}, & 0^{\circ} & <\theta & <90^{\circ} \\ \delta_{L}, & -90^{\circ} & <\theta & <0^{\circ}\end{array}\right.$ 


\section{MRI Methods}

MRI experiments were conducted in a horizontal $7 \mathrm{~T} / 30 \mathrm{~cm}$ AVIII MRI system (Bruker-Biospin Inc., Billerica, MA, USA), equipped with gradients capable of $450 \mathrm{mT} / \mathrm{m}$ amplitude (Resonance Research Inc.). A home-built, transmit-only birdcage volume RF coil, $12 \mathrm{~cm}$ internal diameter, was used for RF excitation. For signal reception, a home-built single surface coil, equipped with dedicated RF preamplifiers, was used. For ASL, a small home-built labeling coil [22] was positioned under the neck of the animal, approximately $2 \mathrm{~cm}$ away from the magnet's isocenter, and connected to the second RF transmit channel of the spectrometer. All coils were equipped with active decoupling circuits to minimize coil-to-coil interferences during the labeling and imaging stages of the experiment and to minimize off-resonance saturation of water in the acquisition region [23].

A localizer tripilot was acquired to position the rat's brain at isocenter. The position of the labeling RF coil with respect to the arteries of interest was verified following acquisition of both sagittal and coronal images of the neck. The labeling position $\Delta z_{0}$ was set to the peak of the longitudinal signal profile generated with the labeling RF coil. Flow-based MR images were acquired using a flowcompensated gradient echo imaging sequence with $\mathrm{TE}=$ $4.3 \mathrm{~ms}$ and $\mathrm{TR}=20 \mathrm{~ms}$. Flow enhancement was achieved by using a large flip angle $\left(90^{\circ}\right)$ for excitation. A single axial slice acquired through the center of the labeling coil and illustrated in Fig. 1b was used to determine the distance of left $\left(\delta_{L}\right)$ and right $\left(\delta_{R}\right)$ carotids from the $x=0$ plane.

Axial ASL perfusion-weighted images were acquired using a spin-echo EPI sequence with the following parameters: $\mathrm{TR}=10,000 \mathrm{~ms}, \mathrm{TE}=28 \mathrm{~ms}, \mathrm{FOV}=32 \times$ $32 \mathrm{~cm}^{2}$, and matrix size $=64 \times 64$. These parameters yielded an in-plane spatial resolution of $500 \mu \mathrm{m}$. A post-labeling delay of $994 \mathrm{~ms}$ was employed to avoid intravascular contamination [24]. A labeling RF pulse of 8,183 ms was applied in the presence of a longitudinal gradient $G_{z}=1 \mathrm{G} / \mathrm{cm}$ at the appropriate labeling frequency offset. For group 1, five 2-mm slices were acquired. For group 2, 15 slices were acquired with $1-\mathrm{mm}$ slice thickness. The contiguous slices were acquired according to a sequential anterior-posterior order to avoid interferences from the acquisition in the magnetization of labeled blood.

Whole-brain MRAs were acquired by using a 2D flow compensated gradient echo sequence with the following parameters: $\mathrm{TR}=30 \mathrm{~ms}, \mathrm{TE}=4.5 \mathrm{~ms}, \mathrm{FOV}=19.2 \times$ $19.2 \mathrm{~mm}^{2}$, matrix $=192 \times 192$. Flow enhancement was achieved by using a $90^{\circ} \mathrm{RF}$ pulse for excitation. A set of 218 axial slices, thickness $=1 \mathrm{~mm}$, each offset along the longitudinal axis by $0.1 \mathrm{~mm}$ with respect to the previous slice, was acquired, resulting in an effective isotropic resolution of $100 \mu \mathrm{m}$. A maximum intensity projection algorithm was used to produce 3D views of the MRA.

Diffusion-weighted images were acquired using an EPI sequence with the following parameters: $\mathrm{TR}=6,000 \mathrm{~ms}, \mathrm{TE}=$ $30 \mathrm{~ms}, \mathrm{FOV}=25.6 \times 25.6 \mathrm{~cm}^{2}$, matrix size $=64 \times 64,15$ slices, slice thickness $=1.5 \mathrm{~mm}, 1$ direction, $b=0$ and $b=1,600 \mathrm{~s} /$ $\mathrm{mm}^{2}$. From the DWI images, apparent diffusion coefficient (ADC) trace maps were calculated using computer software (DPTools, Denis Ducreux, Paris, France). T2-weighted images were acquired at 1 day after MCAO using a relaxation enhancement sequence: $\mathrm{TR} / \mathrm{TE}=12,000 / 74 \mathrm{~ms}$, matrix $=128 \times 128, F O V=25.6 \times 25.6 \mathrm{~mm}^{2}$. All images in the present work are presented in direct coordinates (i.e., right hemisphere on right side of image).

\section{Data Analysis}

Ischemic lesions during occlusion and $1 \mathrm{~h}$ after reperfusion were determined on the ADC maps by using a $23 \%$ reduction in mean contralateral ADC [25]. One day after MCAO, lesions were determined by manual tracing of the hyperintense areas on the T2-weighted images. The corresponding $\mathrm{ADC}$ and $\mathrm{T} 2$ lesion volumes were then calculated by summing the abnormal area and multiplying by the slice thickness. Acquired lesion volumes were corrected for the space occupying effect due to edema as previously described by Gerriets et al. [26].

Quantitative CBF maps were calculated using MATLAB R2009a (The MathWorks, Natick, MA, USA). Control and labeled images were separately averaged and then subtracted. CBF values $(\mathrm{ml} / 100 \mathrm{~g} / \mathrm{min})$ were calculated using the following equation [23]:

$\mathrm{CBF}=\frac{\lambda}{T_{1}} \frac{S_{C}-S_{L}}{S_{L}+(2 \alpha-1) S_{C}}$,

where $\lambda$ is the brain/blood partition coefficient, $T_{1}$ is the longitudinal relaxation time of the brain water magnetization in the absence of perfusion and cross-relaxation, $S_{C}$ is the equilibrium signal intensity, $S_{L}$ is the brain water signal intensity from the labeled image, and $\alpha$ is the labeling efficiency. The labeling efficiency was determined experimentally and the values of $S_{C}$ and $S_{L}$ were measured for all subjects. $\lambda=0.9 \mathrm{ml} / \mathrm{g}$ [27] and $T_{1}=1.75 \mathrm{~s}$ were used in all calculations.

\section{Statistics}

All values and error bars were reported as mean \pm standard deviation. They were compared by multi-factor analysis of variance and post-hoc Bonferroni's test using SYSTAT 122007 (Systat Software, Inc., Chicago, IL, USA). Significance was set at $P<0.05$. 


\section{Results}

\section{Selective ASL Parameters Optimization}

The RF power used for CASL was initially optimized to produce the highest labeling efficiency by observing the signal intensity in the internal carotid artery at different labeling RF amplitudes [22, 28]. Increasing the labeling power causes the signal in the carotid arteries to decrease. This means that the blood flowing through the artery is experiencing a larger flip angle as it passes the labeling coil region. Increasing further the RF power brings back the brightness of the signal from the vessels, which means that the flip angle has passed $90^{\circ}$, and appears positive due to the magnitude mode display of the images. By measuring the signal at the center of the internal carotid arteries, the labeling efficiency was determined and a minimum power of $2 \mathrm{~W}$, defined as the optimal power for all further experiments, was required to achieve the maximum labeling efficiency of $75 \pm 2 \%$ in the neck region.

The selectivity of labeling the semi-hemispheric perfusion territories was optimized by varying the angle $\theta$ of the labeling plane (Fig. 1a). When $\theta=0^{\circ}$, the condition for the regular ASL experiment is achieved and both hemispheres are efficiently labeled, as shown in Fig. 2b. As $\theta$ increases, the perfusion signal in the left hemisphere decreases due to reduced efficiency of labeling of the left circulation. A typical $300 \mathrm{~g}$ rat has the CCAs separated by $\delta=\delta_{L}+\delta_{R} \approx$ $1 \mathrm{~cm}$ (Fig. 1b). Because the excitation profile of our

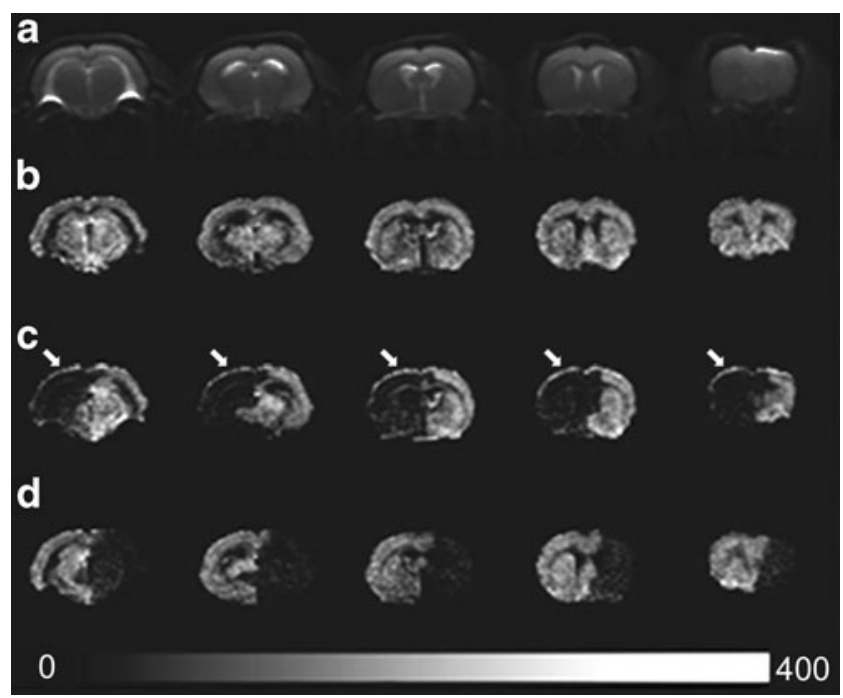

Fig. 2 a Anatomical axial images of a typical SHR rat. b Global perfusion-weighted images acquired using $\theta=0^{\circ}$. c Perfusion images of the right hemisphere, corresponding to the perfusion territory of the right CCA, acquired using $\theta=+60^{\circ}$ and $\mathbf{d}$ of the left hemisphere, corresponding to the perfusion territory of the left CCA, acquired using $\theta=-60^{\circ}$. The color bar expresses the CBF values in $\mathrm{mL} / 100 \mathrm{~g} /$ min. Arrows show the leptomeningeal circulation perfusing the outer cortical layers labeling coil is $E P_{z} \approx 1.5 \mathrm{~cm}$, it is necessary to tilt the labeling plane by $\theta>\tan ^{-1}\left(\frac{\mathrm{EP}_{z}}{2 \cdot \delta}\right)=37^{\circ}$ to start to differentiate the left from the right CCA territories. In preliminary experiments performed using $\theta= \pm 45^{\circ}$, there was noticeable perfusion contrast in the contralateral side $(46 \%$ relative to the ipsilateral side, data not shown), indicating significant labeling of the arterial spins and thus insufficient selectivity to discriminate accurately the left from the right circulation. When $\theta= \pm 60^{\circ}$ was used, perfusion signal on the contralateral side was reduced by up to $77 \%$ relative to the ipsilateral side (Table 1). The use of even larger angles of the labeling plane introduced image artifacts due to the intersection of the labeling gradient with the most posterior brain region. Thus, $\theta= \pm 60^{\circ}$ was used as the optimal tilt angle for all further experiments.

\section{Vascular Territories}

Figure 2a shows axial EPI anatomical images of a typical SHR rat covering most of the brain from posterior to anterior. The corresponding whole-brain CBF maps obtained using $\theta=0^{\circ}$ are shown in Fig. 2b. There was equal sensitivity to perfusion of both cerebral hemispheres, with good gray versus white matter contrast and interesting perfusion heterogeneity in different brain regions likely attributed to regional differences in metabolic rate [29]. To obtain the right perfusion territory as shown in Fig. 2 c, $\theta=$ $+60^{\circ}$ was used. The leptomeningeal circulation perfusing the outer cortical layers is also shown in Fig. 2c (arrows). Likewise, to obtain the left perfusion territory, $\theta=-60^{\circ}$ was used (Fig. 2d). Table 1 lists the calculated mean CBF values obtained at $\theta=0^{\circ}, \theta=-60^{\circ}$, and $\theta=+60^{\circ}$ for WKY and SHR of group 1. Whole-brain $\mathrm{CBF}$ values in left and right hemispheres were identical at $\theta=0^{\circ}(P>0.45)$, indicating uniform performance of the labeling coil. However, use of the tilted labeling plane significantly decreased CBF in the contralateral side, while maintaining $\mathrm{CBF}$ values in the ipsilateral hemisphere $(P<0.01)$. Attenuation of the signal

Table 1 Cerebral blood flow (CBF) values $(\mathrm{mL} / 100 \mathrm{~g} / \mathrm{min})$ obtained at $\theta=0^{\circ},-60^{\circ}$, and $+60^{\circ}$ in Wistar Kyoto (WKY) and spontaneously hypertensive rats (SHR) of group 1

\begin{tabular}{|c|c|c|c|c|c|c|}
\hline & \multicolumn{2}{|l|}{$\theta=0^{\circ}$} & \multicolumn{2}{|l|}{$\theta=-60^{\circ}$} & \multicolumn{2}{|c|}{$\theta=+60^{\circ}$} \\
\hline & Left & Right & $\operatorname{Left}^{\mathrm{a}}$ & Right & Left & Right $^{\mathrm{a}}$ \\
\hline WKY & $86 \pm 17$ & $84 \pm 17$ & $80 \pm 18$ & $32 \pm 6$ & $34 \pm 8$ & $80 \pm 19$ \\
\hline SHR & $147 \pm 25$ & $147 \pm 25$ & $144 \pm 21$ & $38 \pm 9$ & $35 \pm 5$ & $149 \pm 25$ \\
\hline
\end{tabular}

There are no differences in $\mathrm{CBF}$ for left versus right hemispheres when $\theta=0^{\circ}(P>0.45)$

${ }^{\mathrm{a}} \mathrm{CBF}$ values are significantly higher in the labeled $\left(\theta= \pm 60^{\circ}\right)$ versus the non-labeled hemisphere $(P<0.01)$ 
in the contralateral hemisphere ranged from $58 \%$ to $77 \%$, and was sufficient to delineate the perfusion territories of the right and left CCAs in both strains.

\section{Comparison of Vascular Territories with MRAs}

We observed a significant variation in individual vascular territories, both in normotensive and hypertensive rats. As exemplified in Fig. 2, across the brain, the left and right perfusion territories were largely complementary, but not entirely symmetric. To understand whether individual variations in perfusion territories could be attributed to variations of the cerebral arterial vasculature, we compared ASL perfusion maps with arterial MRAs in each subject in group 1.

Figure 3 shows examples of the variability of perfusion territories and arterial angiograms in five subjects. Respective MRAs (left/middle columns) and colorcoded ASL territory maps (right column) highlight the observed dominance of the ACA and the thalamic arteries regions. In Fig. 3a, a WKY rat shows dominance of the right ACA (arrows, right column) and thalamic artery (right dominance - RD), evidenced by an encroachment of the right perfusion territory (green) over the left hemisphere. The corresponding angiogram shows a bigger caliber of the right ACA (Fig. 3a, middle column, arrows). A similar pattern of RD of the right ACA perfusion territory was also found in SHRs (arrows in Fig. 3c, right column). In this case, the corresponding angiogram shows a buttonhole formation of the right ACA [30]. A different and interesting pattern is shown in Fig. $3 \mathrm{~d}$-an alternating leftright dominance (ALRD) of the ACA region of a SHR. The most posterior slices show encroachment of the left perfusion territory onto the right hemisphere (Fig. 3d, right column), whereas the most anterior ones reveal an inverted pattern. The corresponding MRA shows a buttonhole formation of the right ACA, which could explain the dominance found in the most anterior slices, but a not in the posterior ones. However, this inversion of pattern could be justified by small vessels contributing asymmetrically to the left side of the ACA, and by an abnormal fusion of the right and left ACA to form the azygos ACA [30]. Both possibilities are suggestively supported by the MRA in Fig. 3d with the caveat that the MRAs are limited by the spatial resolution of $100 \mu \mathrm{m}$. Other patterns of asymmetry commonly found include a significant contribution from the left ACA to perfusion of the right hemisphere (left dominance-LD), which was observed both in WKY (Fig. 3b) and SHR (Fig. 3e). The respective angiograms show bigger caliber and buttonhole formation of the left ACA. In addition, both rats presented thalamic artery dominance.

Overall, RD-ACA was present in six animals (four WKY rats and two SHRs), whereas LD-ACA was present in another three animals (two WKYs and one SHR). One SHR presented ALRD-ACA and the remaining two SHRs presented no dominance (ND) in the ACA region. Thalamic artery dominance was observed in five subjects (three WKY rats and two SHRs).

\section{Alterations in Vascular Territories following MCA Occlusion}

To investigate changes in perfusion territories following an ischemic insult to the brain, the right MCA was occluded for $1 \mathrm{~h}$ in all 14 rats of group 2. Perfusion territories were evaluated before and during MCAO, and then again $1 \mathrm{~h}$ and 1 day after reperfusion. Table 2 shows the perfusion territories measured at different time points from both left and right cerebral hemispheres of all rats in group 2 . Relative changes in the perfusion territories during ischemia and after reperfusion are also shown. Prior to ischemia, six rats (three WKY and three SHR) presented left dominance of the ACA territory prior to MCAO $(P<$ 0.00002), while the remaining rats (four WKY and four SHR) presented right dominance of the ACA territory $(P<$ $0.00002)$. There were no statistically significant differences in perfusion territories between WKY and SHR animals at any of the time points measured, and thus, data from both strains were combined according to the territorial dominances (Table 2).

Changes in perfusion territories after unilateral MCAO were observed in all 14 rats, regardless of their particular dominance. During ischemia, there was a significant decrease of the right perfusion territory and an increase of the left perfusion territory (Table $2, P<0.0006$ ), indicating a drop of CBF to the ischemic (right) side and the appearance of compensatory collateral flow from the contralesional (left) side. There was no influence of dominance on the reduction in right perfusion territory during ischemia $(-66 \pm$ $14 \%$ in animals with left dominance, $-66 \pm 4 \%$ in animals with right dominance, $P>0.92$ ). However, there was a strong effect of dominance on the increase in left perfusion territory during ischemia. Animals with right dominance presented a larger volume change of the left perfusion territory $(23 \pm 9 \%)$ than animals with left dominance $(7 \pm$ $5 \%, P<0.002)$. After reperfusion, the volume of the right perfusion territory recovered to $-6 \pm 9 \%$ in animals with left dominance prior to the ischemia, not statistically different than the recovery to $-15 \pm 8 \%$ in animals with original right dominance $(P>0.93)$. Meanwhile, the volume of the left territory in animals with left dominance recovered to its original values $(1 \pm 7 \%)$, but remained significantly increased $(17 \pm 12 \%, P<0.002)$ in animals with right dominance. Taken together, the data suggest that, during unilateral ischemia and following reperfusion, the collateral flow in animals with contralesional dominance manifests 
Fig. 3 Comparison of perfusion territories with MRAs. The first column shows MRAs obtained from individual rats. A detailed view of the anterior side of the Circle of Willis (middle column) shows variations (arrows) that may explain the asymmetries observed in the perfusion territory maps (right column, arrows). Green=right perfusion territory; red=left perfusion territory. $L D$ Left dominance, $R D$ right dominance, $A L R D$ alternating left-right dominance, $A C A$ anterior cerebral artery, $B A$ basilar artery, $C C A$ common carotid artery, $E C A$ external carotid artery, ICA internal carotid artery, $P P A$ pterygopalatine artery

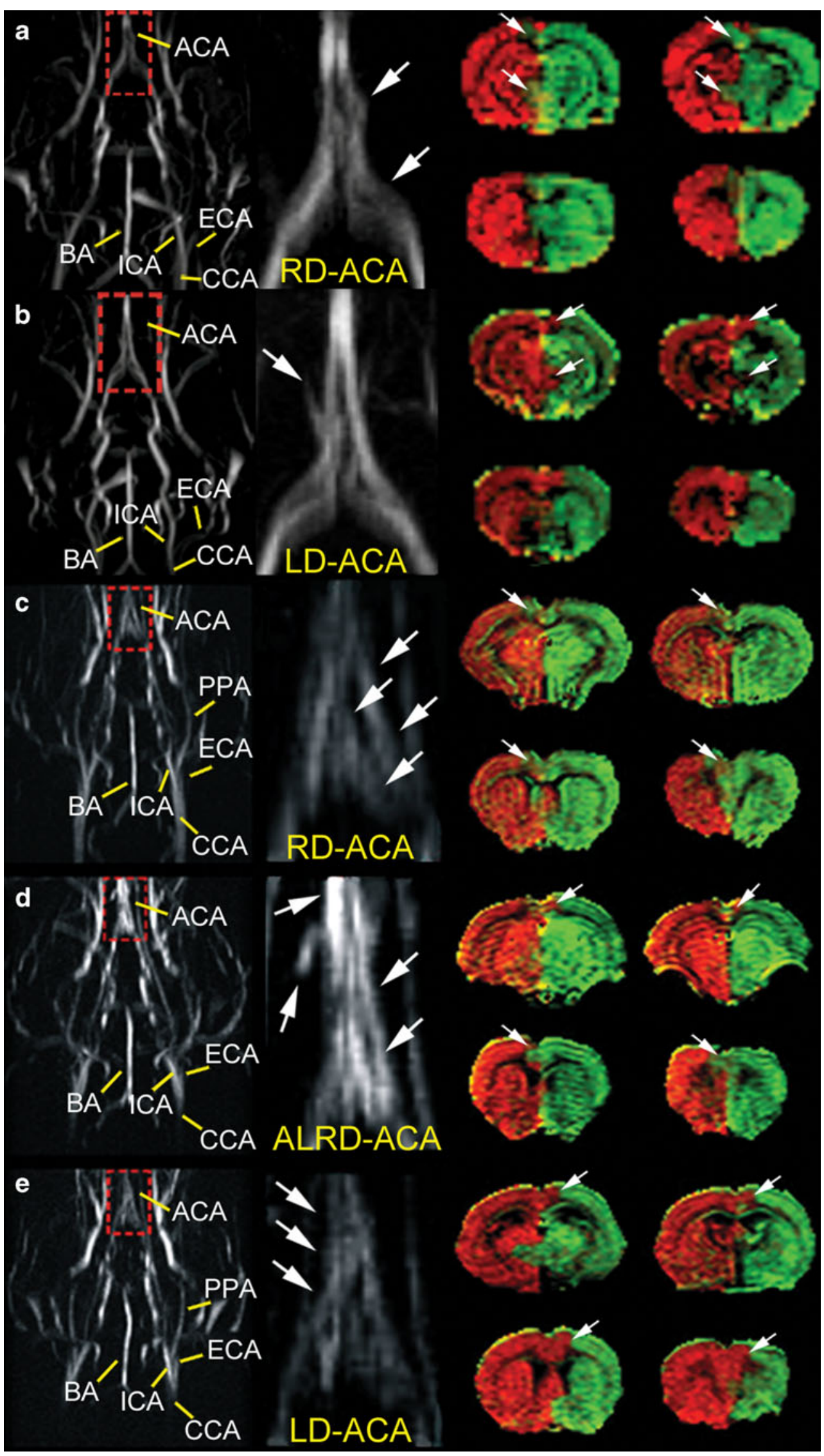


Table 2 Absolute $\left(\mathrm{mm}^{3}\right.$, mean $\left.\pm \mathrm{SD}\right)$ and Relative $(\%$, mean $\pm \mathrm{SD})$ perfusion territories measured at different time points from the left and right cerebral hemispheres of all rats in group $2(n=14)$. Relative changes $(\%$, mean $\pm \mathrm{SD})$ in perfusion territories during ischemia and after reperfusion with respect to the respective volumes prior to ischemia are also shown

Perfusion Territories

\begin{tabular}{|c|c|c|c|c|c|c|}
\hline & \multicolumn{2}{|c|}{ Prior to ischemia } & \multicolumn{2}{|c|}{ During ischemia $^{a}$} & \multicolumn{2}{|c|}{ After reperfusion } \\
\hline \multicolumn{7}{|c|}{ Left dominance prior to ischemia } \\
\hline & Left & Right & Left & Right & Left & Right \\
\hline Absolute & $668 \pm 69^{\mathrm{b}}$ & $562 \pm 39$ & $714 \pm 52^{b}$ & $194 \pm 93$ & $676 \pm 70^{\mathrm{b}}$ & $530 \pm 82$ \\
\hline Relative & $55 \pm 3 \%^{\mathrm{b}}$ & $46 \pm 2 \%$ & $59 \pm 3 \%{ }^{\mathrm{b}}$ & $16 \pm 7 \%$ & $56 \pm 4 \%{ }^{\mathrm{b}}$ & $43 \pm 4 \%$ \\
\hline Changes & & & $7 \pm 5 \%$ & $-66 \pm 14 \%{ }^{\mathrm{e}}$ & $1 \pm 7 \%$ & $-6 \pm 9 \%$ e,f \\
\hline \multicolumn{7}{|c|}{ Right dominance prior to ischemia } \\
\hline & Left & Right & Left & Right & Left & Right \\
\hline Absolute & $533 \pm 53^{\mathrm{b}}$ & $619 \pm 31$ & $654 \pm 58^{\mathrm{b}}$ & $208 \pm 27$ & $616 \pm 28^{\mathrm{b}, \mathrm{c}}$ & $525 \pm 44^{\mathrm{d}}$ \\
\hline Relative & $46 \pm 3 \%^{\mathrm{b}}$ & $54 \pm 3 \%$ & $57 \pm 4 \%{ }^{\mathrm{b}}$ & $18 \pm 3 \%$ & $54 \pm 3 \%$ b,c & $46 \pm 3 \%{ }^{\mathrm{d}}$ \\
\hline Changes & & & $23 \pm 9 \% \mathrm{o}^{\mathrm{g}}$ & $-66 \pm 4 \% 0^{\mathrm{e}}$ & $17 \pm 12^{\mathrm{g}}$ & $-15 \pm 8 \%$ e,f \\
\hline
\end{tabular}

${ }^{a}$ Perfusion territories of either side or dominance during ischemia vs. prior to ischemia or after reperfusion $(P<0.0006)$

${ }^{\mathrm{b}}$ Perfusion territories on left side vs. right side $(P<0.00002)$

${ }^{\mathrm{c}}$ Left perfusion territories after reperfusion vs. before ischemia $(P<0.003)$

${ }^{\mathrm{d}}$ Right perfusion territories after reperfusion vs. before ischemia $(P<0.02)$

${ }^{\mathrm{e}}$ Changes in perfusion territory on right side vs. left side $(P<0.00001)$

${ }^{\mathrm{f}}$ Changes in perfusion territory after reperfusion vs. during ischemia $(P<0.00001)$

${ }^{\mathrm{g}}$ Changes in perfusion territory in right vs. left dominance $(P<0.002)$

itself primarily as an increase in CBF, while animals with ipsilesional dominance show a reversal of dominance and a significant increase in contralesional perfusion territory.

To better illustrate the data on Table 2, Fig. 4 shows the perfusion territory maps of an SHR with a small left dominance of the ACA territory prior to the ischemia (Fig. 4a). The leptomeningeal circulation perfusing the outer cortical layers is also shown in Fig. 4a. During ischemia (Fig. 4b), there was a significant increase in the left dominance of the ACA territory, and development of a left dominance of the territory of the thalamic artery (solid contours in Fig. 4b). There was also an increase in the leptomeningeal circulation on the right side (arrows in Fig. 4b). This increased left dominance of the ACA

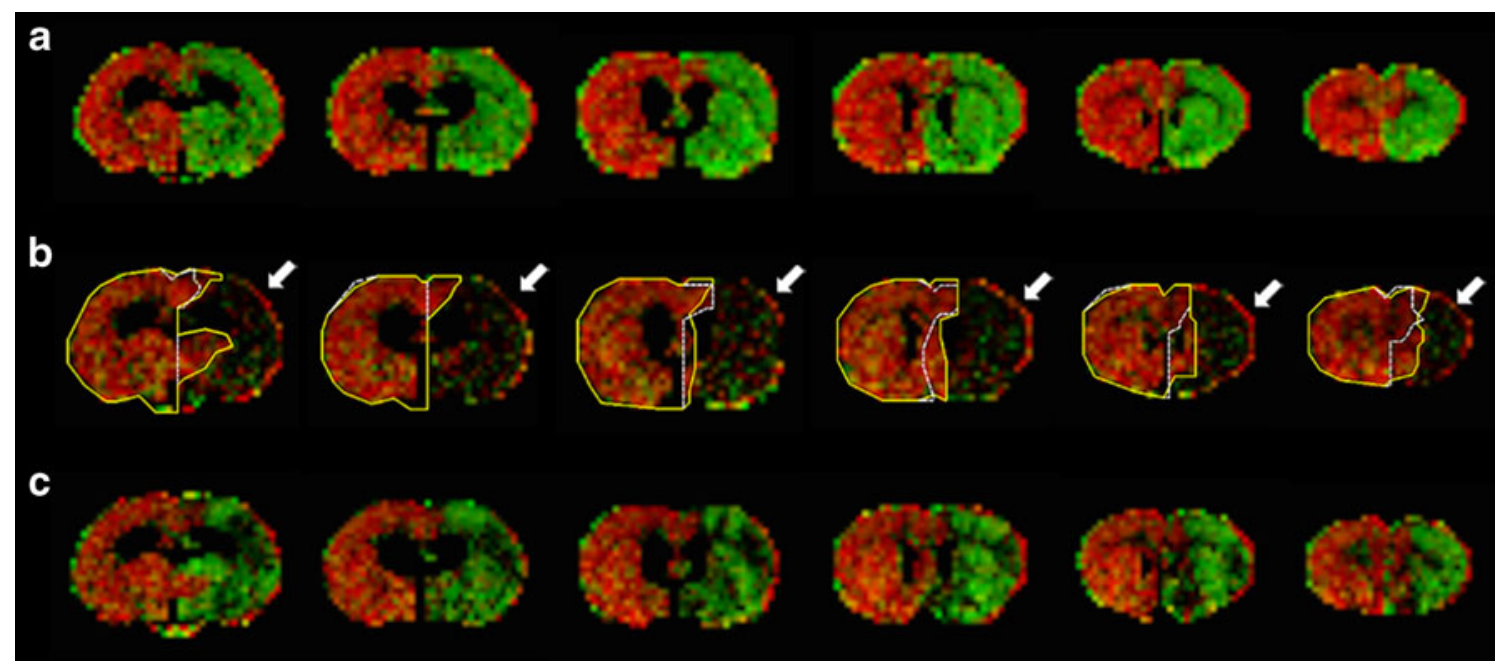

Fig. 4 a Left dominance of the ACA perfusion territory (dashed contours in b) prior to the occlusion of the right middle cerebral artery (MCAO). b During MCAO, there was an increase of the left perfusion territory (solid contours). Notice the significantly decreased perfusion of the right hemisphere, and the increased perfusion through the leptomeningeal circulation (arrows). $\mathbf{c}$ The left dominance of the ACA territory persisted after reperfusion 
territory and the establishment of left dominance of the thalamic artery persisted after reperfusion (Fig. 4c). However, the leptomeningeal circulation was restored to pre-ischemic levels. Across all six rats with left dominance of the perfusion territories, there was an increase of $7 \pm 5 \%$ in the volume of the left territory during MCAO.

The change in perfusion territories following MCAO was even more dramatic for the seven rats presenting right dominance of the ACA territory. In these rats, MCAO caused a switch to left dominance, resulting in a volume increase of $23 \pm 9 \%$ of the left territory. Figure 5 shows the perfusion territory maps of an SHR that presented right dominance of the ACA territory before MCAO (arrows in Fig. 5a). During MCAO, the dominance changed and the left circulation supplied a good portion of the right hemisphere, especially in the most frontal slices (arrows in Fig. 5b). After reperfusion, the left dominance persisted (Fig. 5c). These findings show an immediate response of collateral flow to the ischemic insult in both WKY and SHR, regardless of the presence of hypertension.

Although no differences were observed between normotensive and hypertensive rats regarding increases in left territory volume due to occlusion of the right MCA, the resulting lesion volumes were significantly larger in SHR than in WKY. This could be partly explained by straindependence differences in collateral CBF in the hemispheric region outside the lesion volume. Figure 6 shows there were significant differences in the collateral $\mathrm{CBF}$ values between both strains. In SHR, collateral CBF values supplied by the left circulation, measured in the right hemisphere outside the lesion volume (blue region shown in Fig. 6a), were significantly lower during ischemia, 1 and $24 \mathrm{~h}$ post-reperfusion compared to baseline values mea- sured prior to ischemia (Fig. 6a, $P<0.05$ ). On the other hand, in WKY rats, no differences in collateral CBF values were observed at any of the time points (Fig. 6a). The lower collateral $\mathrm{CBF}$ values in SHR correlated with larger lesion volumes compared to WKY rats (Fig. $6 \mathrm{~b}, P<0.05$ ). Table 3 shows $\mathrm{CBF}$ values calculated in the right hemisphere, within (yellow region in Fig. 6a) and outside (blue region in Fig. 6a) the perfusion-deficient lesion. These findings suggest impairment of collateral development in SHR after ischemic stroke is one of the reasons for the larger lesion volumes observed in hypertensive rats, when compared to normotensive controls (Fig. 6b).

\section{Discussion}

In the present work, we have demonstrated the successful combination of continuous ASL, vascular territory mapping and MRA to perform a comprehensive mapping of the perfusion territories of the major cerebral feeding arteries in rodents. We observed that both WKY and SHR have complementary, yet significantly asymmetric perfusion territories, which correlate well with variations of the Circle of Willis. Furthermore, we observed significant changes in the perfusion territories after temporary ischemic stroke that show the usefulness of CASL to probe the collateral circulation.

Vascular territory mapping is a promising tool for the study of cerebrovascular diseases $[12,13]$. The use of ASL to obtain vascular territories has the advantages of not requiring injections of contrast agents, and of allowing multiple, repetitive measurements to be carried out within a single clinical visit or across several visits. The ASL-based

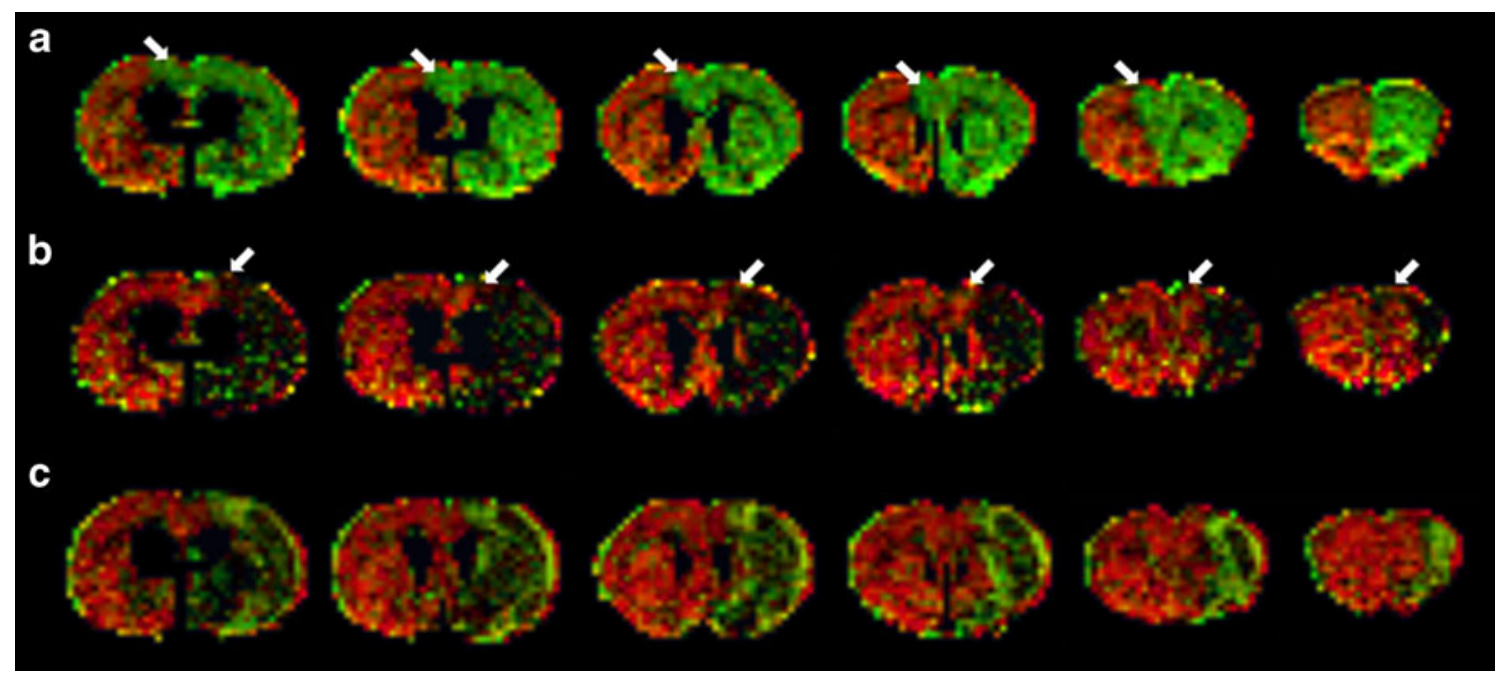

Fig. 5 a Prior to the occlusion of the right middle cerebral artery (MCAO), the rat presented a right dominance of the ACA territory (arrows). b During MCAO, there was a clear change in dominance of the perfusion territory of the ACA (arrows), which persisted $\mathbf{c}$ after reperfusion. Leptomeningeal circulation was also observed in this rat 
Fig. 6 a Collateral CBF values obtained from SHR $(n=7)$ and WKY $(n=7)$ rats within the region outside the ischemic lesion in the right hemisphere supplied by the left circulation (blue ROI in brain image). Unlike their normotensive controls, SHR rats failed to maintain collateral $\mathrm{CBF}$ values during ischemia and at 1 and $24 \mathrm{~h}$ post-reperfusion. b Ischemic lesion volumes measured during MCAO, and at $1 \mathrm{~h}$ and 1 day post-reperfusion. SHR rats showed significantly larger lesion volumes than WKY rats $\left({ }^{*}\right.$ vs. WKY; ${ }^{\text {t }}$ vs. baseline; $P<0.05)$
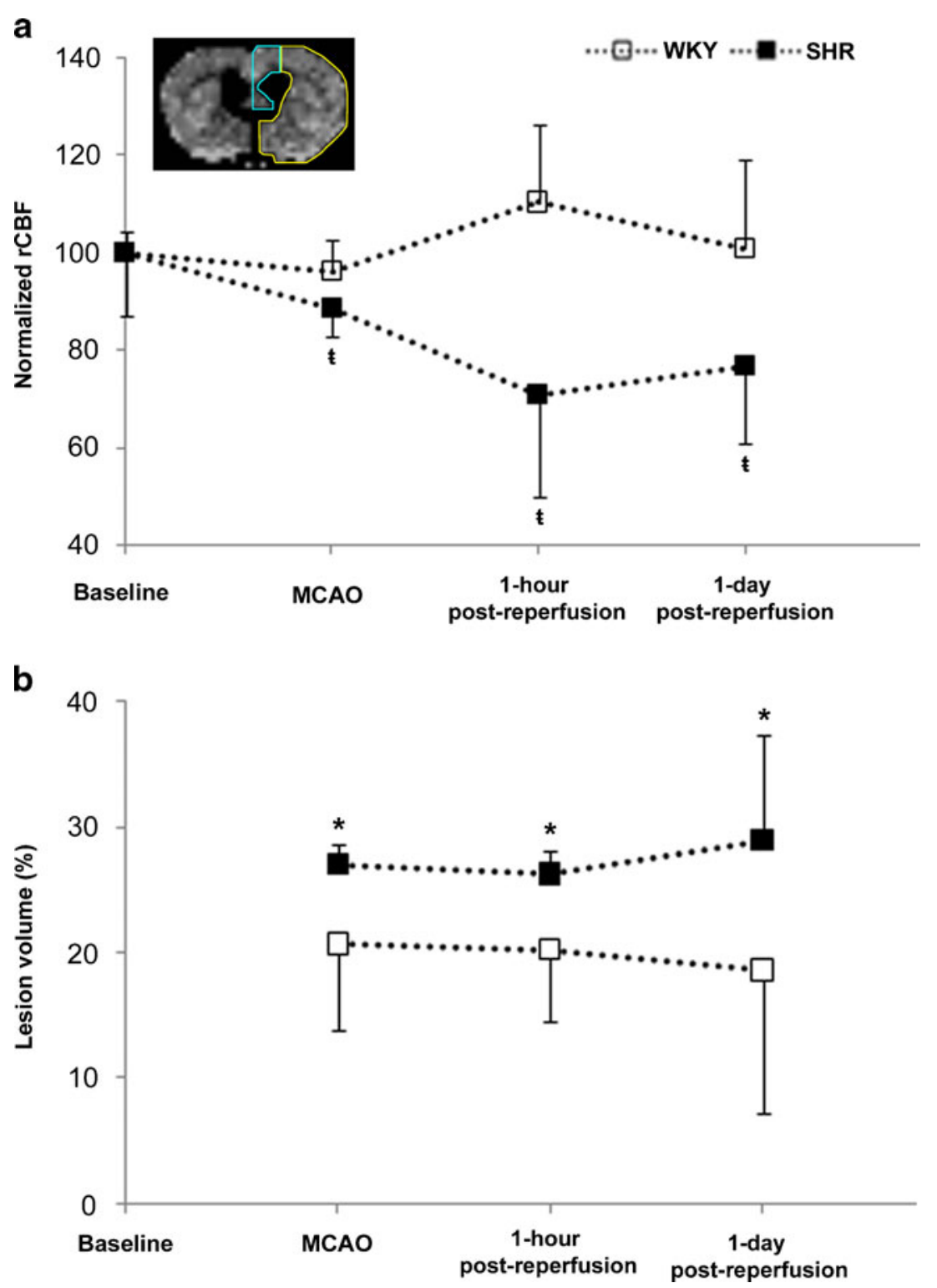

method described herein for determining vascular territories was successfully applied to humans [31]. An important issue to determine the overall quality of these classes of techniques that allow the measurement of vascular territo- ries is the selectivity of the label. The high selectivity of the described method can be verified by mean of the CBF values obtained from the labeled and unlabeled sides. This difference also reflects the high SNR in the selected
Table 3 Absolute $\mathrm{CBF}$ values $(\mathrm{mL} / 100 \mathrm{~g} / \mathrm{min}$, mean $\pm \mathrm{SD})$, measured at different time points from the right cerebral hemisphere of WKY $(n=7)$ and SHR $(n=7)$ rats

\section{$P<0.05$}

\section{${ }^{\mathrm{a}} \mathrm{WKY}$ vs. SHR}

${ }^{b}$ vs. prior to ischemia in WKY

${ }^{c}$ vs. prior to ischemia in SHR

\begin{tabular}{|c|c|c|c|c|}
\hline & Prior to ischemia & During ischemia & After reperfusion & Day 1 \\
\hline \multicolumn{5}{|c|}{ Within the perfusion deficient lesion } \\
\hline WKY & $84 \pm 12$ & $24 \pm 4^{\mathrm{a}, \mathrm{b}}$ & $68 \pm 24$ & $75 \pm 26$ \\
\hline SHR & $97 \pm 11$ & $17 \pm 3^{c}$ & $62 \pm 23^{c}$ & $48 \pm 13^{\mathrm{c}}$ \\
\hline \multicolumn{5}{|c|}{ Outside the perfusion deficient lesion } \\
\hline WKY & $96 \pm 9$ & $93 \pm 6$ & $106 \pm 15^{\mathrm{a}}$ & $97 \pm 18^{\mathrm{a}}$ \\
\hline SHR & $101 \pm 9$ & $89 \pm 6^{\mathrm{c}}$ & $71 \pm 21^{\mathrm{c}}$ & $77 \pm 16^{\mathrm{c}}$ \\
\hline
\end{tabular}


territories. In addition, because the labeling RF coil encompasses both left and right circulation in the neck, good quality images were obtained over the whole brain within the same experimental session.

In agreement with some recent studies in humans [32, 33], we have demonstrated a large variability in flow territories of the anterior circulation in normotensive and hypertensive rats. As shown in Fig. 2, the most common perfusion territories asymmetries observed in the present work were those residing in the territories of the anterior (ACA), the middle (MCA), and the posterior (PCA) cerebral arteries. In addition, we also observed asymmetries in the thalamic artery. These variations in flow territories are likely to be caused by anatomical variants of the COW. However, from the magnetic resonance angiograms shown in Fig. 3 which are limited to an isotropic spatial resolution of $100 \mu \mathrm{m}$, we could only see a correlation of the caliber of the ACA with the regional asymmetries. It is likely that variations of the MCA and PCA are too subtle to be fully resolved with MRA. For example, an interesting pattern was observed in the perfusion territory of the paraventricular thalamic and hypothalamic nuclei (Fig. 3e), but the MRA data could not elucidate any anatomical variance in that region.

All observed territory variants were present in both rat strains. Although the identification of a particular variant does not infer the level of function or vasoreactive response to insult or injury, the ACA right-left dominances observed are of particular interest. In the clinical setting, the anterior communicating artery $(\mathrm{ACoA})$ is present as a bridge between the right and left ACAs, providing a "pressure-stop" between individual hemispheres. Following stroke, this equilibrium is disrupted and compensatory flow develops towards the low-pressure side, for example, from the left ACA to the right $\mathrm{ACA}$ in the case of a right $\mathrm{M} 1$ occlusion. In contrast, there is no ACoA present in the rat, making the flow patterns dependent on the ACA variant present (ND, RD, LD, ALRD). Because the ACA is the major artery providing collateral flow to the MCA territory, understanding the collateral development in branches of the ACA can play an important role in elucidating variations in the extent of the ischemic region after an insult.

In the present work, we chose the well-established suture model to produce proximal occlusion of the right MCA [20]. Other occlusion models, including the distal MCA occlusion [34], could have been used, but have a disadvantage of requiring craniotomy, which could compromise the quality of the MRI data. Notwithstanding, identical procedures were carried out in both SHR and WKY strains. The resulting lesion volumes were significantly larger in the hypertensive rats. Chronic hypertension is known to cause vascular remodeling in the form of hypertrophy and eutrophy, which leads to stiffening of the blood vessels and causes impairment of the mechanisms of autoregulation and cerebrovascular reactivity [35]. The present data suggest that impairment of collateral development in SHR after ischemic stroke is one of the reasons for the larger lesion volumes observed in hypertensive rats. As described in Liesbeskind [1], the main arteries of the Circle of Willis constitute the primary collateral circulation, while the leptomeningeal vessels make the secondary collateral circulation. Our CASL technique was sensitive enough to distinguish both forms of collateral circulation. While the largest variance in perfusion territories occurred in vessels of the COW, the present data suggest that the leptomeningeal circulation is also recruited during ischemia and may have an important role in protecting the brain against ischemic damage [36]. Further experiments are necessary to investigate the role of the leptomeningeal vessels in experimental stroke.

In both rat strains, collateral flow was observed after occlusion of the MCA, regardless the dominance of the perfusion territory before the occlusion. However, the particular dominance plays an important role in determining how collateral flow is elicited. Our data suggest that collateral flow in animals with ipsilesional dominance involves primarily safeguarding local CBF values with small changes in contralesional perfusion territory, while animals with ipsilesional dominance show a reversal of dominance and a substantial increase in contralesional perfusion territory. As shown in Table 3 and Fig. 6, the maintenance of sustained collateral $\mathrm{CBF}$ values in the region immediately adjacent to the perfusion-deficient region is critical to keeping the lesion volume relatively small. Hypertension impairs maintenance of collateral flow, leading to larger lesion volumes. This result is in agreement with previous studies that reported suppressed collateral development, after arterial occlusion, in SHR relative to age-matched WKY [37, 38]. Therefore, ASL vascular territory maps and angiograms may be used in future studies to assess the collateral flow development at different time points during acute stroke as well as chronically, to further understand its correlation with arterial occlusion outcome, and also to elucidate the effects of risk factors, such as hypertension.

In conclusion, we combined a conventional CASL sequence, a dedicated labeling coil, and the proper placement and direction of the labeling plane to assess and characterize the vasculature of rats. Interesting results were reported regarding the correlation between the perfusion territories and the anatomical formations of the COW. Moreover, alterations in perfusion territories after artery occlusion could be evaluated, confirming that the present method may enable better understanding of the cerebral hemodynamics. 
Acknowledgments The authors acknowledge the excellent technical assistance of Mrs. Xianfeng (Lisa) Zhang. This research was supported by the Intramural Research Program of the NIH, NINDS (Alan P. Koretsky, Scientific Director), and FAPESP (2006/05706-5, 2003/13399-7, 2005/56663-1).

\section{References}

1. Liebeskind DS. Collateral circulation. Stroke. 2003;34(9):2279-84.

2. Bang OY, Saver JL, Kim SJ, Kim GM, Chung CS, Ovbiagele B, et al. Collateral flow predicts response to endovascular therapy for acute ischemic stroke. Stroke. 2011;42(3):693-9.

3. Maas MB, Lev MH, Ay H, Singhal AB, Greer DM, Smith WS, et al. Collateral vessels on $\mathrm{CT}$ angiography predict outcome in acute ischemic stroke. Stroke. 2009;40(9):3001-5.

4. Bang OY, Saver JL, Buck BH, Alger JR, Starkman S, Ovbiagele $\mathrm{B}$, et al. Impact of collateral flow on tissue fate in acute ischaemic stroke. J Neurol Neurosurg Psychiatry. 2008;79(6):625-9.

5. Liebeskind DS. Collaterals in acute stroke: beyond the clot. Neuroimaging Clin N Am. 2005;15(3):553-73. x.

6. Detre JA, Leigh JS, Williams DS, Koretsky AP. Perfusion imaging. Magn Reson Med. 1992;23(1):37-45.

7. Golay X, Hendrikse J, Lim TC. Perfusion imaging using arterial spin labeling. Top Magn Reson Imaging. 2004;15(1):10-27.

8. Williams DS, Detre JA, Leigh JS, Koretsky AP. Magnetic resonance imaging of perfusion using spin inversion of arterial water. Proc Natl Acad Sci U S A. 1992;89(1):212-6.

9. Hoffman JM, Coleman RE. Perfusion quantitation using positron emission tomography. Invest Radiol. 1992;27 Suppl 2:S22-6.

10. Villringer A, Rosen BR, Belliveau JW, Ackerman JL, Lauffer RB, Buxton RB, et al. Dynamic imaging with lanthanide chelates in normal brain: contrast due to magnetic susceptibility effects. Magn Reson Med. 1988;6(2):164-74.

11. Detre JA, Zhang W, Roberts DA, Silva AC, Williams DS, Grandis DJ, et al. Tissue specific perfusion imaging using arterial spin labeling. NMR Biomed. 1994;7(1-2):75-82.

12. Paiva FF, Tannus A, Silva AC. Measurement of cerebral perfusion territories using arterial spin labelling. NMR Biomed. 2007;20 (7):633-42.

13. van Laar PJ, van der Grond J, Hendrikse J. Brain perfusion territory imaging: methods and clinical applications of selective arterial spin-labeling MR imaging. Radiology. 2008;246(2):354-64.

14. Chng SM, Petersen ET, Zimine I, Sitoh YY, Lim CC, Golay X. Territorial arterial spin labeling in the assessment of collateral circulation: comparison with digital subtraction angiography. Stroke. 2008;39(12):3248-54.

15. Jones CE, Wolf RL, Detre JA, Das B, Saha PK, Wang J, et al. Structural MRI of carotid artery atherosclerotic lesion burden and characterization of hemispheric cerebral blood flow before and after carotid endarterectomy. NMR Biomed. 2006;19(2):198-208.

16. Hendrikse J, Petersen ET, Cheze A, Chng SM, Venketasubramanian N, Golay X. Relation between cerebral perfusion territories and location of cerebral infarcts. Stroke. 2009;40(5):1617-22.

17. van Laar PJ, Hendrikse J, Klijn CJ, Kappelle LJ, van Osch MJ, van der Grond J. Symptomatic carotid artery occlusion: flow territories of major brain-feeding arteries. Radiology. 2007;242(2):526-34.

18. Zaharchuk G, Do HM, Marks MP, Rosenberg J, Moseley ME, Steinberg GK. Arterial spin-labeling MRI can identify the presence and intensity of collateral perfusion in patients with Moyamoya disease. Stroke. 2011;42(9):2485-91.

19. Amenta F, Di Tullio MA, Tomassoni D. Arterial hypertension and brain damage-evidence from animal models (review). Clin Exp Hypertens. 2003;25(6):359-80.
20. Longa EZ, Weinstein PR, Carlson S, Cummins R. Reversible middle cerebral artery occlusion without craniectomy in rats. Stroke. 1989;20(1):84-91.

21. Maccotta L, Detre JA, Alsop DC. The efficiency of adiabatic inversion for perfusion imaging by arterial spin labeling. NMR Biomed. 1997;10(4-5):216-21.

22. Silva AC, Zhang W, Williams DS, Koretsky AP. Multi-slice MRI of rat brain perfusion during amphetamine stimulation using arterial spin labeling. Magn Reson Med. 1995;33(2):209-14.

23. Zhang W, Silva AC, Williams DS, Koretsky AP. NMR measurement of perfusion using arterial spin labeling without saturation of macromolecular spins. Magn Reson Med. 1995;33(3):370-6.

24. Alsop DC, Detre JA. Reduced transit-time sensitivity in noninvasive magnetic resonance imaging of human cerebral blood flow. J Cereb Blood Flow Metab. 1996;16(6):1236-49.

25. McCabe C, Gallagher L, Gsell W, Graham D, Dominiczak AF, Macrae IM. Differences in the evolution of the ischemic penumbra in stroke-prone spontaneously hypertensive and Wistar-Kyoto rats. Stroke. 2009;40(12):3864-8.

26. Gerriets T, Stolz E, Walberer M, Muller C, Kluge A, Bachmann A, et al. Noninvasive quantification of brain edema and the spaceoccupying effect in rat stroke models using magnetic resonance imaging. Stroke. 2004;35(2):566-71.

27. Herscovitch P, Raichle ME. What is the correct value for the brain-blood partition coefficient for water? J Cereb Blood Flow Metab. 1985;5(1):65-9.

28. Zhang W, Williams DS, Koretsky AP. Measurement of rat brain perfusion by NMR using spin labeling of arterial water: in vivo determination of the degree of spin labeling. Magn Reson Med. 1993;29(3):416-21.

29. Sokoloff L, Reivich M, Kennedy C, Des Rosiers MH, Patlak CS, Pettigrew KD, et al. The $[14 \mathrm{C}]$ deoxyglucose method for the measurement of local cerebral glucose utilization: theory, procedure, and normal values in the conscious and anesthetized albino rat. J Neurochem. 1977;28(5):897-916.

30. Brown JO. The morphology of circulus arteriosus cerebri in rats. Anat Rec. 1966;156(1):99-106.

31. Paiva FF, Tannus A, Talagala SL, Silva AC. Arterial spin labeling of cerebral perfusion territories using a separate labeling coil. J Magn Reson Imaging. 2008;27(5):970-7.

32. Hendrikse J, van der Grond J, Lu H, van Zijl PC, Golay X. Flow territory mapping of the cerebral arteries with regional perfusion MRI. Stroke. 2004;35(4):882-7.

33. van Laar PJ, Hendrikse J, Golay X, Lu H, van Osch MJ, van der Grond J. In vivo flow territory mapping of major brain feeding arteries. NeuroImage. 2006;29(1):136-44.

34. Brint S, Jacewicz M, Kiessling M, Tanabe J, Pulsinelli W. Focal brain ischemia in the rat: methods for reproducible neocortical infarction using tandem occlusion of the distal middle cerebral and ipsilateral common carotid arteries. J Cereb Blood Flow Metab. 1988;8(4):474-85.

35. Iadecola C, Davisson RL. Hypertension and cerebrovascular dysfunction. Cell Metab. 2008;7(6):476-84.

36. Brozici M, van der Zwan A, Hillen B. Anatomy and functionality of leptomeningeal anastomoses: a review. Stroke. 2003;34 (11):2750-62.

37. Omura-Matsuoka E, Yagita Y, Sasaki T, Terasaki Y, Oyama $\mathrm{N}$, Sugiyama $\mathrm{Y}$, et al. Hypertension impairs leptomeningeal collateral growth after common carotid artery occlusion: restoration by antihypertensive treatment. J Neurosci Res. 2011;89(1):108-16.

38. Tuttle JL, Sanders BM, Burkhart HM, Fath SW, Kerr KA, Watson $\mathrm{WC}$, et al. Impaired collateral artery development in spontaneously hypertensive rats. Microcirculation. 2002;9(5):343-51. 